\title{
Characterization of Segmented HPGe Detectors Using Pulse Shape Comparison Methods
}

\author{
F.C.L. Crespi ${ }^{\text {a,b }}$, V. Vandone ${ }^{a, b}$, F. Camera ${ }^{a, b}$, S. Brambilla ${ }^{b}$, B. Million ${ }^{b}$, S. Riboldi ${ }^{a, b}$, \\ O. Wieland ${ }^{\text {b }}$ \\ ${ }^{a}$ Physics Department, University of Milan, Via Celoria 16, 20133 Milano, Italy \\ ${ }^{b}$ INFN Sezione di Milano, Via Celoria 16, 20133 Milano, Italy
}

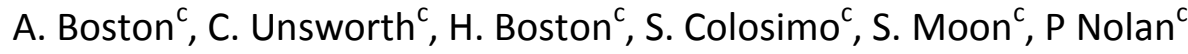 \\ ${ }^{c}$ Department of Physics, University of Liverpool, Liverpool L69 3BX, UK
}

Gamma ray tracking arrays like AGATA [1] and GRETA [2] will be used in experiments with radioactive ion beams [3]. These arrays are composed of large volume 36 -fold segmented HPGe detectors. The gamma interaction points must be localized with a precision $<5 \mathrm{~mm}$ by the combined use of detector electrode segmentation and Pulse Shape Analysis (PSA). In order to properly perform the PSA it is fundamental to precisely $(\sim \mathrm{mm})$ characterize the HPGe detector position response (i.e. associate the signal shape to each position inside the detector volume). This kind of information is presently extracted both experimentally $[4,5]$ and numerically $[6,7,8]$. However an important limitation of the standard experimental techniques is the extremely long time required for the measurements.

In previous works $[9,10]$ a new detector characterization method, the Pulse Shape Comparison based Scan (PSCS), has been presented. This technique enormously decreases the time duration of the measurements, allowing a full scan of a large volume $\left(\sim 140 \mathrm{~cm}^{3}\right)$ HPGe segmented detector in less than 1 week.

In this work we show the results of the PSCS applied for the first time to a large volume segmented HPGe detector from the AGATA array. The experimental signals have been taken with an asymmetric AGATA crystal by the Liverpool University gamma spectroscopy group, using the experimental set-up described in [5] and a 137Cs collimated source. Three different datasets have been used: singles scan of the detector front face ("S_1", figure 1a), singles scan of the detector side ("S_2", figure 1b) and coincidence scan ("C", figure 1c). The latter is obtained applying the standard technique [5] for extracting the detector signal shape associated to a determined $X, Y, Z$ position.

In the first part of the work a consistency check between dataset " $C$ " and "S_1" has been performed. This test consisted in selecting different signals from the dataset " $C$ " (associated to a determined $X, Y, Z$ position), extracting then from dataset "S_1" the signals that have the same shape. The $X^{\prime}, Y$ ' coordinates (i. e. collimator position) of the selected events in dataset "S_1" must then correspond to $X, Y$ of the event in dataset " $C$ ". For all the different $X, Y$ positions selected it has been always found good agreement between the spatial coordinates. An example of these results is reported in figure 2.

In the second part of the work the PSCS method has been applied: using datasets "S_1" and "S_2" (i.e. two datasets with the collimation lines perpendicular one in respect to the other) it has been possible to extract the position response of two segments in the front part of the detector, with a $\sim 1 \mathrm{~mm}$ precision. This feature is important since with the standard "coincidence" method the detector scan in Z direction is limited to very few points. We are presently performing the comparison of the signals obtained with the PSCS and those of the standard coincidence scan. This latter part of the work is still ongoing and it will constitute the final validation of the method. The variation of the signal shape as a function of the gamma interaction position (inside the 2 scanned segments) will be shown. 

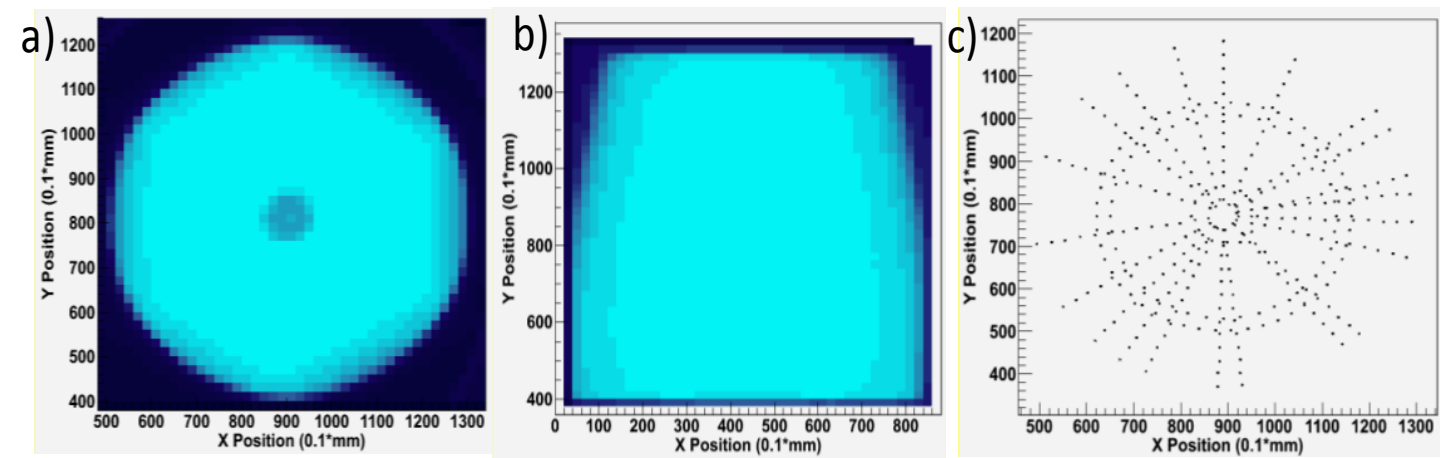

Figure 1. a) Number of signals collected for each $X, Y$ collimator position. The collimator is swept across the front face of the detector in $1 \mathrm{~mm}$ steps. The decrease in statistics due to the presence of the central hole can be noted. b) Same as a), the collimator is now swept across the side of the detector. These are the two datasets used for the PSCS. c) X,Y profile of the positions scanned with the standard coincidence method.
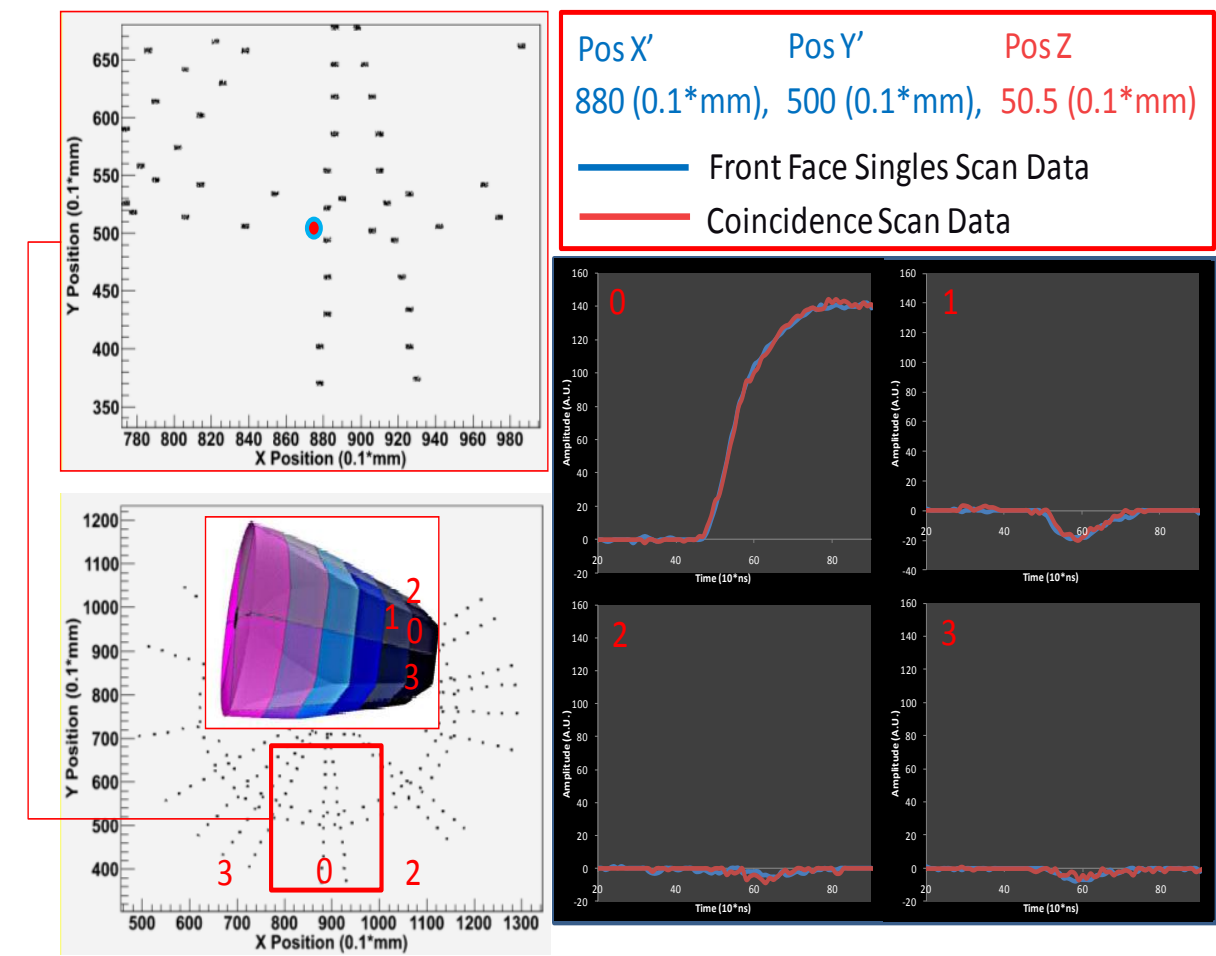

Figure 2. The red signal shapes are extracted with the standard coincidence technique (dataset " $(\mathrm{C}$ "); the red dot in the upper left panel indicates the $X, Y$ position associated to the signal shapes while the $Z$ is indicated in red color in the upper right panel. The blue shape is extracted from dataset "S_1" using a $\chi^{2}$ comparison. As can be seen the $X$ ', $Y$ " coordinates precisely match the $X, Y$. The red numbers indicate the detector segments associated to the signals.

[1] AGATA Technical Proposal, edited by J. Gerl and W. Korten, September 2001. available at http://www-w2k.gsi.de/agata/ and D. Bazzacco, Nucl. Phys. A 746 (2004) 248 C

[2] C.W. Beausang, Nucl. Instr. and Meth. B 204 (2003), p. 666 and M. A. Deleplanque, et al., Nucl. Inst. and Meth. A 430 (1999) 292

[3] R. Lieder, et al., Prog. Part. Nucl. Phys. Vol 46 (2001) 399 and H.J. Wollersheim, Nucl. Instr. and Meth. A 537 (2005), p. 637.

[4] K. Vetter, et al., Nucl. Inst. and Meth. A 452 (2000) 223-238

[5] A. Boston, et al., Nucl. Inst. and Meth. B 261 (2007) 1098-1102

[6] P. Medina et al., A Simple Method for the Characterization of HPGe Detectors, IMTC 2004, Como, Italy, website:

http://mgs2005.in2p3.fr/Mgs.php

[7] M. Schlarb, R. Gernhäuser, R. Krücken, Simulation and Real-Time Analysis of Pulse Shapes from HPGe Detectors, GSI Scientific Report 2008, p. 232.

[8] B. Bruyneel, et al., Nucl. Inst. and Meth. A 608 (2009) 99

[9] F.C.L. Crespi, Invited talk at the 2008 Nuclear Science Symposium, IEEE, Dresden, Germany

[10] F.C.L. Crespi, et al, Nucl. Instr. and Meth. A 593 (2008), p. 440. 